\section{Transfusion Medicine and Hemotherapy}

\author{
Review Article . Übersichtsarbeit
}

Transfus Med Hemother 2007;34:20-32

DOI: $\underline{10.1159 / 000098179}$
Received: December 18, 2006

Accepted: December 19, 2006

Published online: January 18, 2007

\title{
Diagnostic Methods for Platelet Function Analysis
}

\author{
Mariam Klouche \\ Bremer Zentrum für Laboratoriumsmedizin und LADR GmbH Medizinisches Versorgungszentrum Bremen, Germany
}

\author{
Key Words \\ Platelet function analysis . Flow cytometry . \\ Aggregometry · ROTEM - Microparticles . \\ Platelet proteome
}

\section{Summary}

Platelets play a key role in the regulation of haemostasis, clot stability and retraction, vascular repair, and exert an increasingly recognised function in host defence. Conversely, platelets are also implied in many pathophysiological processes, such as thrombosis, haemorrhage, inflammation, atherogenesis, tumour growth and metastasis. Thus quantitative and qualitative platelet alterations have been associated with distinct clinical diseases, including cardiovascular diseases, diabetes, tumour metastasis and sepsis, and consequently different diagnostic platelet markers are correlated with the prognosis and severity of these diseases. While primary platelet functional abnormalities are very rare diseases, acquired platelet function disorders are rather frequent and occur in the context of many diseases and in association with several drugs. Furthermore, the introduction of a great variety of different anti-platelet agents leads to iatrogenic states of therapeutically reduced platelet function. This review provides an overview of the methodological principles of platelet function analysis, the currently available diagnostic devices, the application and suitability for the diagnosis of different platelet function disorders and the restrictions of each method, to promote an optimal selection of diagnostic tests.

\section{Schlüsselwörter}

Thrombozytenfunktionsanalyse - Durchflusszytometrie · Aggregometrie · ROTEM · Mikropartikel ·

Thrombozytenproteom

\section{Zusammenfassung}

Thrombozyten spielen eine zentrale Rolle in der Regulation der Hämostase, der Stabilität und Retraktionsfähigkeit von Thromben sowie der Gefäßregeneration und üben darüber hinaus eine wichtige Funktion in der Immunabwehr aus. Umgekehrt sind Thrombozyten ebenfalls an vielen pathophysiologisch relevanten Prozessen beteiligt, wie beispielsweise an der Thrombose, der Blutungsneigung, der Entzündung, der Atherogenese, dem Tumorwachstum und der Metastasierung. Quantitative und qualitative Veränderungen der Thrombozyten wurden mit unterschiedlichen Erkrankungen assoziiert. So sind bei kardiovaskulären Erkrankungen, Diabetes, Tumormetastasen und Sepsis unterschiedliche diagnostische thrombozytäre Marker mit der Prognose der Erkrankung assoziiert. Während die primären funktionellen Thrombozytendefekte extrem seltene Erkrankungen sind, kommen die erworbenen Störungen der Thrombozytenfunktion im Kontext zahlreicher Erkrankungen und im Zusammenhang mit der Einnahme vieler Medikamente vor. Daneben führten die gerade in den letzten Jahren eingeführten unterschiedlichen antithrombozytären Therapien zu iatrogen vermittelten therapeutischen Thrombozytenfunktionsstörungen. In dieser Übersichtsarbeit wird ein Überblick über die methodischen Prinzipien der Thrombozytenfunktionsanalyse und die gegenwärtig verfügbaren Analysatoren gegeben, die Anwendung und Eignung der Tests bei unterschiedlichen Thrombozytenfunktionsstörungen erörtert sowie die Vorbehalte und Einschränkungen der Methoden dargestellt, um eine optimale Auswahl der am besten geeigneten Testmethoden für die jeweilige Fragestellung zu fördern.

\begin{tabular}{ll}
\hline KARGER & (c) 2007 S. Karger GmbH, Freiburg \\
$\begin{array}{l}\text { Fax +49 7614 5207 14 } \\
\begin{array}{l}\text { E-mail Information@Karger.de } \\
\text { www.karger.com }\end{array}\end{array}$ & $\begin{array}{l}\text { Accessible online at: } \\
\text { www.karger.com/tmh }\end{array}$ \\
&
\end{tabular}

Prof. Dr. med. Mariam Klouche

Bremer Zentrum für Laboratoriumsmedizin und

LADR GmbH Medizinisches Versorgungszentrum

Friedrich-Karl Straße 22, 28205 Bremen, Germany

Tel. +49 421 4307-233, Fax -534

E-mail mariam.klouche@laborzentrum-bremen.de 


\section{Introduction}

Platelets have a central function in the regulation of haemostasis, clot stability and retraction, vascular tone and repair as well as in host defence. Consequently, platelet dysfunction has a major impact on many pathophysiological processes, such as thrombosis, haemorrhage, inflammation, atherogenesis and cardiovascular disease, tumour growth and metastasis as well as anti-microbial host defence $[1,2]$.

Human platelets are anucleate small cells $(2-3 \mu \mathrm{m}$ measured by microscopy; $0.5-1 \mu \mathrm{m}$ measured in solution by flow cytometry), with nucleic acid remnants from megacaryocytes, which have been recently recognised to contain unique intracytoplasmatic transcriptionally active signal-depending premRNA splicing loci [3]. The usual life-span is 7-10 days, and the normal concentration in blood ranges from 150 to $400 \times$ $10^{9} / 1$. Besides mitochondria, lysosomes and endoplasmatic reticulum, platelets contain specific granules, the $\alpha$-granula, and the dense bodies (fig. 1). Platelet $\alpha$-granules store platelet and plasmatic coagulation activation factors such as von Willebrand factor (vWF), multimerin, thrombospondin-1, fibrinogen and coagulation factors but also serve as a reservoir for $\mathrm{IgE}$ and chemokines such as platelet factor-4 (PF-4), betathromboglobulin ( $\beta$-TG) and RANTES [4-6]. Degranulation in platelet concentrates may cause clinically relevant transfusion reactions upon platelet transfusion [7]. Dense bodies contain adenosine diphosphate (ADP), serotonin, and $\mathrm{Ca}^{2+}$. In fact, PF-4 and $\beta$-TG were the first chemokines discovered in platelets, but their functional roles in host defence and their involvement in the recruitment and activation of leucocytes as well as in the regulation of tissue repair became clear only recently and cornered the term 'thrombokines'. The surfaceconnected open canalicular system (OCS) allows a huge amplification of the platelet surface and permits the activation, the endo- as well as the exocytosis of active mediators by platelets.

Normal platelet function in haemostasis involves structured adhesion, activation, de-granulation of stored contents, homophilic aggregation (platelet-platelet), heterophilic aggregation (platelet-leucocyte), coagulation factor surface binding, fibrinogen binding, clot formation and retraction (fig. 2). Formation of the platelet clot requires fine-tuned interaction with plasmatic coagulation factors, particularly with vWF, fibrinogen as well as with subendothelial matrix proteins. The initial accumulation of platelets, shape change and formation of a platelet monolayer on spread vWF supports thrombin generation and ultimately platelet aggregation. The key platelet surface receptors mediating the central interaction steps include platelet vWF binding by glycoprotein $(\mathrm{Gp}) \mathrm{Ib} / \mathrm{IX} / \mathrm{V}$ and integrin $a_{I I} \beta_{1}$, as well as platelet collagen binding by GpVI and GpIIb/IIIa (CD41/CD61; integrin aIIb $\beta_{3}$ ) (fig. 2, left). Further extension of the platelet aggregate is critically dependent on expression of members of the superfamily of G-protein coupled receptors, which bind to the classic locally generated and released platelet activators ADP, thrombin, and thromboxane A2 (TxA2), and amplify the reaction. The complete devolution results in irreversible platelet activation and aggregation (fig. 2, right). Platelet function in host defence includes the re-

Fig. 1. Platelet structure and composition.

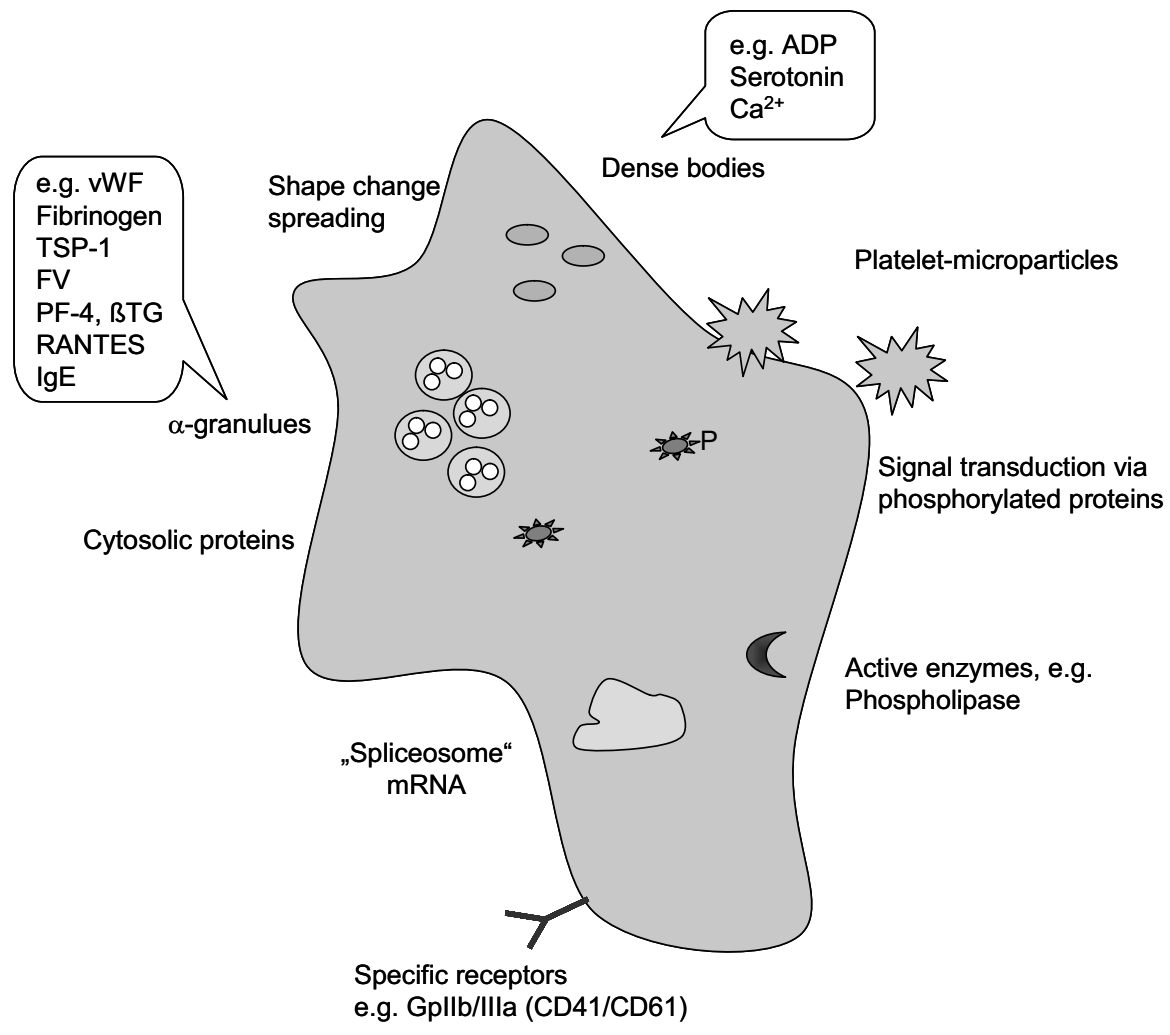


Fig. 2. Stages of platelet activation and vascular clot formation. Sequential interaction between platelets and subendothelial matrix (e.g. collagen), vWF, as well as exogenous activation, and endogenous amplification of the activation reaction by homotypic local interactions between platelets.

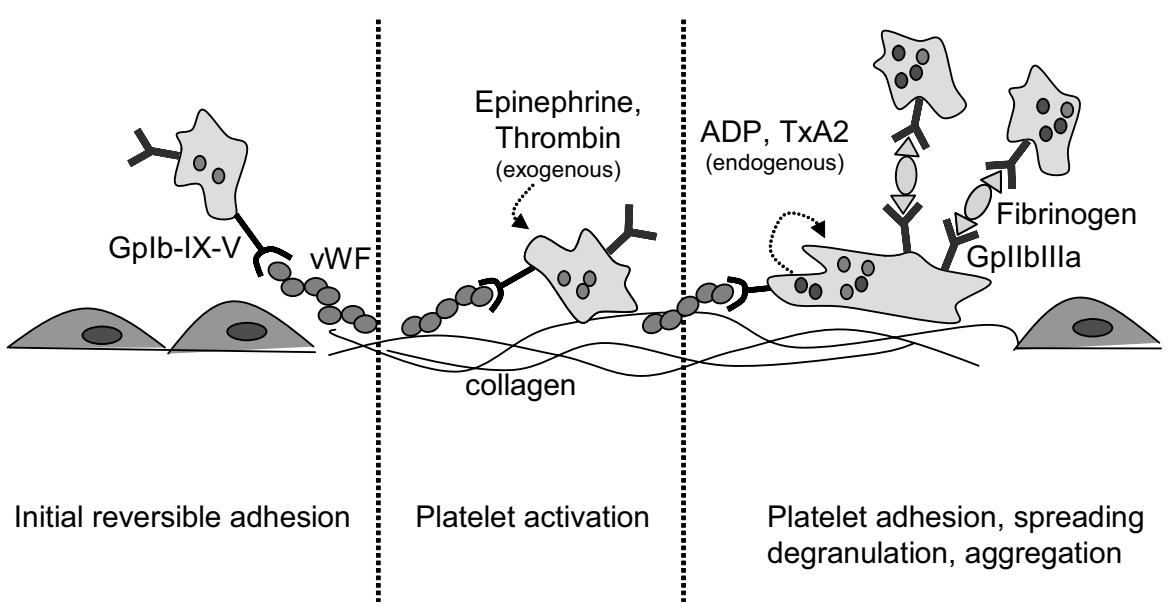

Platelet adhesion, spreading versible release of chemokines and $\mathrm{IgE}$ from intracellular granules, allowing the rechargement of the granule content by active uptake via surface receptors and different ways of endocytosis partly via the OCS $[5,8]$.

The inherited platelet $\mathrm{Gp}$ defects constitute a group of very rare disorders of combined structural and functional platelet disturbances [1, 9-11]. The common denominator of these defects is an impaired platelet adhesion, activation and clot formation at sites of vascular injury. Thus, the diagnosis is usually based on the simultaneous occurrence of one or several of the following findings: prolonged skin bleeding time, presence of a small number of very large platelets (macrothrombocytopenia), defective ristocetin-induced platelet aggregation, markedly reduced prothrombin consumption and low or absent expression of platelet surface Gp complexes. Typically, not a single platelet abnormality is present, but rather a combination of different functional defects prevails, which complicates diagnosis. In addition, other inherited genetic disorders, particularly diseases with defective lysosomal function such as Hermansky-Pudlak syndrome and Chediak-Higashi disease, are associated with a bleeding diathesis resulting from platelet storage pool deficiency.

Acquired secondary or treatment-associated platelet function deficiencies by far outnumber the primary inherited platelet function deficiencies. A number of different disorders, including liver function disorders, uraemia, different myeloproliferative disorders and dysproteinaemias, as well as diseases associated with anti-platelet antibodies such as immune thrombocytopenia (ITP), and acquired von Willebrand's disease may be associated with platelet function abnormalities. Similarly, a broad array of drugs affects platelet function (table 1).

At present, no single platelet function test is suitable to address all distinct steps of platelet activation for the differential diagnosis of the variety of different primary and secondary platelet function disorders. Most platelet function tests are performed in specialised coagulation laboratories which usually provide a broad portfolio of tests addressing distinct functional aspects as well as global coagulation tests as applied for patient testing $[12,13]$ and blood product quality measures in transfusion medicine [14]. Recently, many simple platelet function tests have become available in closed-system analysers, which principally may also be used in a point-of-care setting. Outside the specialised laboratory, these systems are mainly applied in emergency settings to evaluate the bleeding risk, and to monitor anti-platelet therapy. For these systems, particularly when used by non-expertised personnel outside the specialised laboratory, pre-analytical measures, validation, quality control and local standardisation of the procedure become major issues to ascertain reliability and reproducibility of the subtle platelet function tests. An overview of the currently available and evolving methods to analyse platelet function and their diagnostic profile including critical reservations are outlined for each analytical test principle. Monitoring of directed anti-platelet therapy is beyond the scope of this review.

\section{Quantitative Platelet Analysis}

As a basis for identification of platelet disorders, platelet numbers and basic morphology are usually evaluated. Although hereditary functional platelet disorders often show normal platelet numbers, they are not infrequently associated with thrombocytopenia. Primary thrombocytosis is a rare acquired chronic disorder of the bone marrow which can occur at any age and may present with similar symptoms as functional platelet disorders, also including thromboembolic and haemorrhagic events and transformation into myelofibrosis or acute leukaemia. Recently, a new a mutation in the JAK2 gene (V617F) was identified in up to $50 \%$ of the patients, which is involved in the pathogenesis of this disease [15].

Principle and use: Platelets are routinely enumerated in automatic haematology analysers, which usually allow detection of pathologically elevated (thrombocytosis) or reduced (thrombocytopenia) platelet numbers, as well as crude morphologic abnormalities. The standard haematology analysers perform 
Table 1. Drug classes affecting platelet function*

\begin{tabular}{ll}
\hline Drug class & Drugs/agent \\
\hline $\begin{array}{l}\text { Directed anti-platelet therapeutics } \\
\text { Cycloxygenase inhibitors }\end{array}$ & $\begin{array}{l}\text { acetylsalicylic acid (e.g. aspirin) } \\
\text { cox2 inhibitors (e.g. celecoxib) }\end{array}$ \\
ADP receptor antagonists & $\begin{array}{l}\text { thienopyridines (e.g. clopidogrel, } \\
\text { ticlopidin) }\end{array}$ \\
GpIIb/IIIa antagonists & $\begin{array}{l}\text { tirofiban } \\
\text { abciximab }\end{array}$ \\
Phosphodiesterase inhibitors & dipyridamol \\
\hline
\end{tabular}

$\begin{array}{cl}\begin{array}{l}\text { Drugs affecting platelet function } \\ \text { Phosphodiesterase inhibitors }\end{array} & \begin{array}{l}\text { number } \\ \text { theophillin } \\ \text { caffeine } \\ \text { vinca alkaloids (e.g. vincristin) }\end{array} \\ \begin{array}{cl}\text { Inhibitors of prostaglandin } \\ \text { metabolism }\end{array} & \begin{array}{l}\text { NSAIDs (e.g. ibuprofen, indometacin) } \\ \text { furosemide }\end{array} \\ \begin{array}{cl}\text { Substances with affinity to lipid } \\ \text { (e.g. penicillin, ampicillin) }\end{array} \\ \begin{array}{c}\text { membranes antibiotics } \\ \text { lidihypertensives (e.g. propanolol) }\end{array} \\ \begin{array}{l}\text { liduction of anti-platelet } \\ \text { antibodies or antibodies } \\ \text { against platelet mediators }\end{array} & \begin{array}{l}\text { anti-infective drugs (e.g. quinine) } \\ \text { anti-fungal drugs (e.g. amphotericine b) } \\ \text { antibiotics (e.g. rifampicin, vancomycin) }\end{array}\end{array}$

NSAID $=$ non-steroidal anti-inflammatory drugs.

*This table provides an overview of important and frequently used drug classes, each with typical examples, but does not attempt completeness.

full blood counts, including platelet counts by using optical (e.g. Sysmex XE 2100, Sysmex; Advia 120, Bayer; Cell-Dyn 4000, Abbott), impedance (XE 2100, Sysmex; LH750, Beckman Coulter) and/or immunological methods (e.g. Cell-Dyn 4000, Abbott). The diagnosis of essential thrombocythaemia is based upon elevated platelet counts, the frequent presence of morphologic and functional alterations and characteristic bone marrow alterations.

Caveats: While quantification of platelets used to be inaccurate in severe thrombocytopenia $(<20,000 / \mu \mathrm{l})$ and typically the platelet count was overestimated $[16,17]$, a linear determination of platelet numbers down to $1,000 / \mu \mathrm{l}$ is ascertained by the actual generation of haematology analysers. EDTA-induced pseudo-thrombocytopenia has to be excluded. In functional platelet abnormalities, bleeding or thrombotic complications of different severity often occur in the presence of normal platelet numbers. Conversely, upon detection of thrombocytosis, other myeloproliferative disorders have to be excluded, which may also be accompanied by increased platelet counts but usually do not show morphologic alterations. Furthermore, both cytoreductive and/or thromboreductive therapy (e.g. with anagrelide) are associated with qualitative and quantitative alterations of platelets; thus secondary effects due to medication may interfere with proper diagnosis of qualita- tive or quantitative platelet disorders. Of note, the platelet count is gender-dependent, being higher in women than in men, and shows no correlation with the thrombopoietin concentration which is generally lower in women [18].

\section{Estimation of Platelet Turnover}

The number of reticulated platelets, which represent the most recently released platelets with a high remaining RNA content, has been introduced as a marker reflecting thrombopoiesis and platelet turnover [19] and may be used as a means of platelet regeneration capacity [20].

Principle and use: Reticulated platelets can be quantified by flow cytometry directly from whole blood without manipulation. The methodologies used are based on staining of the nucleic acid content of platelets by fluorescent stains, e.g. thiazole orange as platelet mRNA stain, after identification of the platelets either by forward and side scatter; or by the use of specific platelet-surface Gp antibodies (e.g. monoclonal anti$\mathrm{Gp}$ antibodies, such as CD61). Thiazole orange is a fluorescent dye which crosses platelet membranes and binds intracellular RNA. At present, only some routine haematology analysers (e.g. Sysmex XE 2100) have the capacity to detect reticulated platelets. Quantification of reticulated platelets is useful for the differential diagnosis and for the analysis of platelet kinetics and can be employed as a useful screening test to differentiate thrombocytopenia with high or low thrombopoietic activity [21, 22]. The assay is suitable to predict the time of platelet recovery and to optimise platelet transfusion decisions. In fact, reticulated platelets have been successfully employed to manage the platelet transfusion requirement during chemotherapy [23]. In addition, circulating reticulated platelets serve as prognostic markers in ischaemic vascular disease [24].

Caveats: Currently reticulated platelets are measured with different methods and protocols, and a standardization of the procedure is still lacking.

\section{Morphologic Examination of Platelets and Mean Platelet Volume}

Platelet size, the presence of giant platelets and more subtle morphologic alterations, e.g. the absence of platelet granules, may provide important diagnostic clues to distinct platelet function defects. Although measurement of the mean platelet volume (MPV) has been available since the 1970s, its relationship with the platelet count and its clinical use as an indicator of platelet activation has only recently been recognised $[25$, 26].

Principle and use: Basic platelet morphology and size can be evaluated by most standard haematology analysers. The MPV usually varies from 7 to $11 \mathrm{fl}$. Automated analysers are gener- 
ally capable to detect platelet aggregates as well as giant platelets, but not infrequently these abnormalities are overlooked. Detection of a pathological MPV, which can easily been detected in several haematology analysers, can add to the diagnosis of the most common forms of inherited macrothrombocytopenia, including Bernard-Soulier syndrome and MYH9-related disease (MYH9-RD) [26]. In thrombocytopenia of unknown origin, the MPV serves as a predictive marker for bone marrow involvement [27]. Additional information may be obtained by standard May-Grünwald-Giemsa-stained peripheral blood smears, allowing the identification of i) platelets lacking in azurophilic granules that are typical of gray platelet syndrome (GPS); ii) red blood cell anisopoikilocytosis and microcytosis, which are present in X-linked thrombocytopenias caused by mutations of GATA-1; and iii) light-blue inclusions in the cytoplasm of polymorphonuclear leucocytes (Döhle-like bodies) that are often detected in MYH9-RD. Some rare platelet disorders, including MYH9-RD or NMMHC-IIA (heavy chain of non-muscle myosin IIA), may be confirmed by the use of specific antibodies in immunocytochemical analysis of peripheral blood films. Finally, electron microscopy allows ultrastructural evaluation of platelets which is diagnostic in platelet granular defects as well as in several giant platelet disorders.

Caveats: Since the MPV is unstable over time and further influenced by the anticoagulant used, the time to analysis is critical.

\section{Basal Platelet Receptor Status and Granule Content}

The basal platelet receptor status together with the determination of the platelet granule composition and content constitute important screening parameters for the detection of inherited platelet disorders.

Principle and use: Quantification of platelet surface glycoprotein receptor density is usually performed using a set of specific monoclonal antibodies labelled with different fluorescent dyes to allow parallel determination by multiparametric flow cytometry [28-30]. Platelet granule deficiency can accurately be determined using the flow cytometric quantification of granule markers on platelet membranes which regularly appear upon platelet activation, provided that the integrity of the granules is given. Integrity of platelet degranulation is usually analysed by flow cytometry, e.g. using P-selectin (CD62P) as marker of platelet $\alpha$-granular degranulation or CD63 as a marker of the integrity of the lysosomal release reaction [31]. Platelet receptor defects and deficiencies of platelet glycoprotein expression can be easily determined, allowing the diagnosis of Glanzmann's thrombasthenia or Bernard-Soulier disease. Besides the pathognomonic absence of the fibrinogen receptor in Glanzmann's thrombasthenia, an impairment of granule release can be detected, which is proportional to the residual expression of platelet GpIIb/IIIa (CD41/CD61). In the GPS the virtual absence of $\alpha$-granules in bone marrow megakaryocytes and circulating platelets is manifested as the absence or severe reduction of the $\alpha$-granule release reaction.

Caveat: Flow cytometric determination of the basal receptor status can reliably be performed using stabilised blood while for all assays involving activation fresh blood is required (see below).

\section{Determination and Quantification of Platelet Activation Markers}

The composition and function of platelet surface receptors as well as the dynamics of platelet activation markers including the estimation of receptor-bound ligands, of the platelet degranulation reaction and of cellular interactions and interactions with components of the plasmatic coagulation system constitute important elements of the platelet activation cascade. Release of platelet activation markers from the dense bodies and/or $\alpha$-granules reflects in vivo platelet activation.

Principle and use: Classically, release of the typical granular markers serotonin (dense bodies) as well as $\beta$-TG and PF-4 ( $\alpha$-granules) were detected in peripheral blood as a measure of the release of platelet granule contents and reflect in vivo platelet activation. The original gold standard assay to detect platelet activation was the detection of serotonin release from pre-labelled platelets, which was classically performed by very demanding methodologies, involving $\left[{ }^{14} \mathrm{C}\right]$ serotonin labelling, high-performance liquid chromatography, and enzyme-linked immunosorbent assays for $\beta$-TG and PF-4.

In vivo platelet activation as well as in vitro reactivity to different agonists such as ADP, epinephrine and thrombin can most accurately and reliably be measured by whole blood flow cytometry [32]. Typical readouts include activation-dependent conformational changes of the activated GpIIb/IIIa receptor (CD41/CD61), which can be detected by PAC1 binding to the exposed fibrinogen binding site, platelet P-selectin (CD62P) expression before and after stimulation or expression of activation markers such as CD63 or CD40L. Moreover, conformational changes of the platelet integrin receptor for fibrinogen binding GpIIb/IIIa can be used to detect platelet activation and receptor recruitment [33]. Activated platelets form heterotypic aggregates with monocytes and neutrophils via engagement of platelet surface P-selectin (CD62P) with leucocyte surface P-selectin glycoprotein ligand-1 (PSGL-1). This engagement induces surface expression of tissue factor and activation of surface Mac-1 (CD11b/CD18) on leucocytes. Upon activation, monocyte surface Mac-1 may bind coagulation factor Xa (FXa) and/or fibrinogen. Moreover, determination of platelet-leucocyte engagement-dependent surface binding of tissue factor, FXa and fibrinogen can be used as a measure of platelet procoagulant activity [34]. In addition, flow cytometric analyses of platelet-derived microparticle formation are available [28-30]. Evidence of platelet activity and hyperreac- 
Table 2. Clinical applications of flow cytometric platelet function tests

Diagnosis of disease

Diagnosis of inherited/specific platelet diseases

- Detection and quantification of platelet receptors

- e.g. Glanzmann's thrombasthenia, Bernard Soulier syndrome, Storage-pool disease

- Characterisation of platelet granule integrity

Detection of in vivo platelet activation

- Detection of activation-dependent conformational changes of platelet receptors (e.g. GpIIb/IIIa, exposure of fibrinogen-binding site) by monoclonal antibodies

- Expression of activation-dependent mediators and receptors (e.g. P-selectin/ CD62P(a-granules), serotonin (dense bodies), CD63 (lysosomes), CD40L

- Comparison of the platelet activation profile before and after exposure to platelet agonists (e.g. ADP, epinephrine)

- Quantification of platelet-platelet and/ or platelet-leukocyte aggregates

- Detection of platelet microparticles

Platelet procoagulant activity

- Binding of coagulation factors

Evaluation of thrombopoiesis and regenerative capacity

- Quantification of reticulated platelets

Workup of thrombocytopaenia

- Detection of platelet-associated immunoglobulins, e.g. exclusion of immune-medited thrombocytopaenia

- Detection of specific anti-platelet antibodies, e.g. anti-HPA-1a

Platelet signal transduction

- Quantification of activation-dependent intracellular protein phosphorylation and/ or calcium-fluxes

- Detection of platelet receptor recruitment and interaction by FRET

Monitoring of treatment

Evaluation of anti-platelet activity Aspirin monitoring and response

Cyclooxygenase inhibitor monitoring

Anti-GpIIb/IIIa treatment (e.g. tirofiban, abciximab)

ADP receptor antagonists (e.g. thienopyridines such as clopidogrel, ticlopidin)

tivity which may contribute to a prothrombotic state can be accurately measured. Similarly, dynamic alterations of the serotonin content can be detected by flow cytometry [35]. Parallel identification of platelets (e.g. by CD41 surface staining), the serotonin content (e.g. by specific anti-serotonin intracytoplasmic staining) and platelet activation (e.g. by annexin $\mathrm{V}$ binding) is employed in multiparameteric flow cytometry. Thereby, flow cytometry is useful to monitor treatment with GpIIb/IIIa inhibitors such as tirofiban which impairs platelet granule release. Platelet activation markers correlate with intraoperative blood loss, and the use of volatile anaesthetics $[13,36,37]$. The value of platelet activation markers as indicators for the prothrombotic risk is outlined in a further review of this series [38]. The minimal manipulation required for flow cytometry allows detection of platelet function in the physio- logic milieu. Only very small amounts of blood are required $(\sim 1 \mu \mathrm{l})$, and the technique can be standardised. Finally, flow cytometry is extremely sensitive, allowing the identification of as low as $1 \%$ of activated platelets. An overview of the broad clinical applications of flow cytometry in platelet function testing is outlined in table 2.

Caveats: Due to the fragility of the degranulation markers, direct measurements of the release of serotonin, $\beta$-TG or PF-4 were prone to artefacts. Analysis of platelet degranulation in platelet-rich plasma (PRP) is prone to artificial activation and degranulation due to the separation process. Thus whole blood flow cytometry is preferable. For dynamic analysis of platelet activation, only fresh blood can reliably be analysed to exclude ex vivo platelet activation while the basal receptor status as well as the identification of in vivo platelet activation can reliably be performed using stabilised blood (e.g. UKNEQUAS or Cyfix, PD Dr. Andreas Ruf, Karlsruhe) [39]. Due to the sensitivity of flow cytometry, changes in the availability of $\mathrm{Ca}^{2+}$ as a result of the use of different anticoagulants may severely influence dynamic results of in vitro platelet activation tests. Some flow cytometric assays require laborious sample preparation and the use of a set of several rather expensive fluorescent-labelled monoclonal antibodies.

\section{Evaluation of Platelet Adhesion}

Platelet adhesion to exposed extracellular matrix, other cell types or vWF is a central early event in the platelet activation cascade. Thus assays have been developed to analyse platelet adhesion capacity to adhesive surfaces.

Principle and use: Platelet adhesive capacity can be evaluated in plates with adhesive surfaces, such as polystyrol, or surfaces coated with extracellular matrix components under high shear rates $\left(1800 \mathrm{~s}^{-1}\right)$. Currently, fully automated (IMPACT ${ }^{\circledR}$, Diamed) and partially automated (e.g. cone and plate analyser (CPA)) devices are available which evaluate adhesion and aggregation concomitantly. After reaction, platelets are stained, and adhesion as well as the number of aggregates formed are quantified using either a software, allowing to determine i) percent adhesion of the available surface, ii) average size and iii) distribution, or the reaction may be observed microscopically. Mean time of analysis is $10 \mathrm{~min}$, and only relatively small amounts of blood $(200 \mu \mathrm{l})$ are required for the test. The test is suited to detect platelet adhesion defects, e.g. Glanzmann's thrombasthenia, defects/deficiencies of platelet GpIIb/IIIa, GpIb, and afibrinogenaemia.

Caveats: Currently limited experience exists with the test. Differences in adhesion capacity are observed with several functional platelet disorders, particularly when distinct adhesive surfaces are employed. Apparently, adhesion to plastic surfaces is not universally suited to evaluate platelet adhesion capacity and has restrictions, e.g. in the diagnosis of thrombasthenia. 

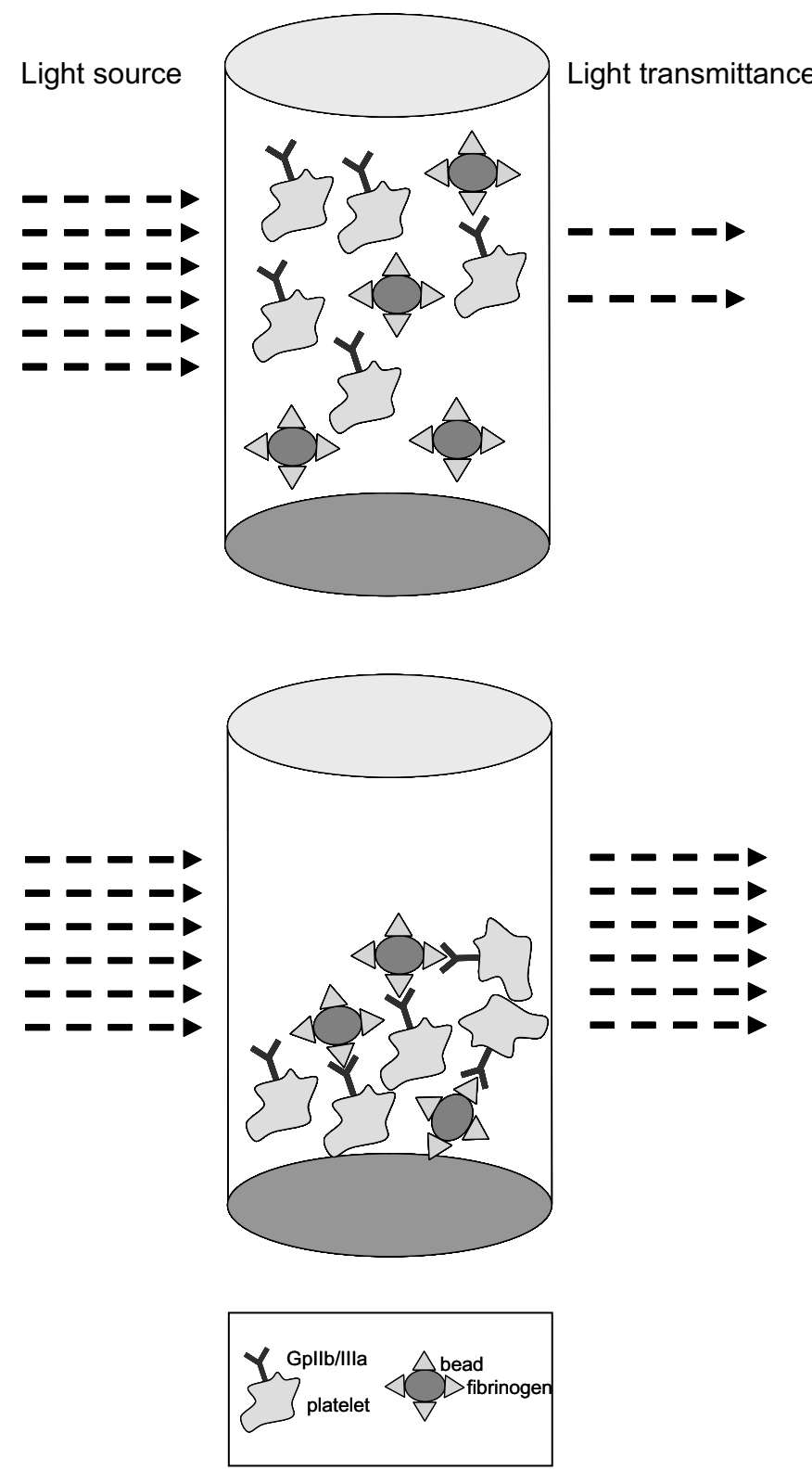

Fig. 3. Principle of turbidimetric platelet aggregation tests. Platelets are incubated with fibrinogen-coated beads (triangles) in the presence of platelets agonists, e.g. ADP. The resulting specific binding to the fibrinogen receptor (GpIIb/IIIa) on platelets leads to agglutination of the beads, which can be measured as increased light-transmittance through the reaction chamber. The increase in light transmittance is proportional to the platelet aggregation formed. Aggregation is reduced in states of diminished platelet activation capacity, e.g. with GpIIb/IIIa deficiency, or in the presence of pharmacologic receptor blockade by GpIIb/IIIa antagonists.

\section{Analysis of Platelet Aggregation}

The traditional platelet function tests encompass the in vitro platelet aggregation, which is performed today using diverse modifications of the original method described by Born et al. more than 40 years ago, as well as the in vivo bleeding time measurement, which together still constitute the most widely used platelet function tests.

Principle and use: In vitro platelet aggregation in PRP is most frequently analysed by the classic Born's method, but may also be evaluated in whole blood. Typically, analysis of spontaneous platelet aggregation by stirring is compared with the induced aggregation by using a panel of different agonists including thrombin, ADP, collagen, epinephrine, ristocetin, arachidonic acid, thromboxane analogue, epinephrine and ristocetin. Each agonist selected for analysis should be evaluated for two different concentrations. The formation of platelet aggregates is proportional to the increase of light transmission which is classically measured using luminometry or impedance technology. Aggregometry is suited to detect functional platelet defects, such as Glanzmann's thrombasthenia or Bernard-Soulier syndrome, and by using ristocetin as a cofactor can be employed for the diagnosis of von Willebrand's disease.

Recently, a turbidimetric method was introduced, measuring platelet aggregation in solution (Ultegra-RPFA ${ }^{\circledR}$, Accumetrics). This test now is available as a point-of-care device (VerifyNow ${ }^{\circledR}$, Accumetrics). The rate of platelet aggregate formation with fibrinogen-coated beads in the presence of platelet activators is determined as the proportional increase of light transmittance (fig. 3). The test is simple to perform and does not require processing of the blood, but requires relatively expensive non-reusable disposables. Since a closed system is used, it is not usually possible to select different platelet activators nor different concentrations for platelet function analysis. However, recently modified cartridges have been employed to evaluate aspirin responsiveness [40].

Assays measuring platelet aggregation using cell counting techniques are available as a rapid $(<10 \mathrm{~min})$, simple whole blood aggregation test (Plateletworks ${ }^{\circledR}$, Helena Biosciences) which requires no sample preparation, and has been shown to correlate well with conventional aggregometry [41, 42]. Agonist-induced platelet aggregation is compared to a control sample without activation, and the degree of platelet aggregation is measured as the difference in platelet counts. The test is rather flexible, allowing platelet activation with different agonists, e.g. with ADP or collagen reagent tubes.

By aggregometry, the different phases of platelet activation and aggregation may be evaluated by analysing i) the slope, which equals the rate of aggregate formation, and ii) the maximal amplitude, which represents the total percentage of aggregates in a given time. Thus, aggregometry gives valuable information in a variety of functional platelet disorders. Aggregometry combined with luminometry is suited to measure platelet aggregation in parallel with the granular release reaction. Caveats: Since aggregometry determines platelet aggregate formation usually in solution in PRP under non-physiological low shear conditions, the test principle does not represent primary haemostasis accurately. Moreover, since the levels and cut-offs of aggregation depend heavily on the respective con- 
ditions of PRP generation, activator setup and local specificities, the analysis of patient's platelet response should preferably be compared to a freshly drawn sample of a normal control. Particularly in the PRP-based transmittance aggregometry methods, care has to be taken to avoid pre-activation of the platelets. Aggregometry has a low specificity, and the methods lack standardisation. In the presence of severe thrombocytopenia, aggregometry cannot be performed. The clinical significance of remote abnormalities in aggregation is questionable. Also for the newer developments of whole blood aggregometry, a limited use for monitoring of aspirinrelated platelet defects has been demonstrated [42]. Enhanced spontaneous in vitro platelet aggregation and/or enhanced platelet response to ristocetin is suggestive of platelet-type and type-2B von Willebrand's disease but requires mixing studies for differential diagnosis.

Although this method has been successful in measuring the aggregation of platelets in a GpIIb/IIIa-dependent manner, it has several limitations, including poor reproducibility, high sample volume, requirement of a sample preparation, length of assay time, requirement for a skilled technician and cost.

\section{Platelet Clot Formation and Stability}

Clot formation and retraction are central final steps of platelet activation, which are also dependent on plasma coagulation and fibrinolysis. Platelets form the major constituent of thrombi which have important physiologic functions in primary haemostasis but also play a crucial role in the pathogenesis of ischaemia, e.g. in coronary syndromes. Clot retraction is a thrombin-dependent, platelet-mediated contraction of the cellular clot mass

Principle and use: Traditionally, thrombelastography (TEG), which has been available for more than 50 years, was used as a global test to analyse the integrity of platelet activation, platelet clot formation, plasmatic coagulation and fibrinolysis / clot lysis [43]. This assay involves whole blood or re-calcified plasma, in which agonists are added to activate platelets. The ensuing clot formation can then be visualised in the oscillating cup by the resulting alteration of the movement of a fine needle. TEG provides broad information on clot formation, including i) the lag time until clot formation starts, as a measure of the thrombin activity, ii) the clotting rate, iii) the maximal amplitude, as a means of clot strength, and iv) the rate of amplitude reduction, as a means of clot lysis (fig. 4). TEG is performed in $30 \mathrm{~min}$ but requires expert interpretation of the resulting thrombelastogramms. Recently, the technology was further developed as the Thrombelastograph PlateletMapping System (Haemoscope Corporation), which is based on the development of a weak platelet clot in the presence of reptilase and FXIII, which upon addition of platelet agonists (e.g. ADP) allows very sensitive detection of alterations of platelet function, particularly the presence of platelet inhibitors. Simi-

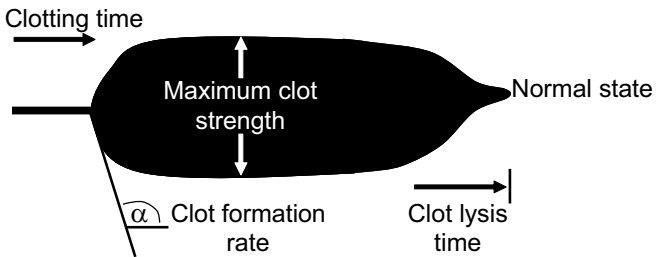

b

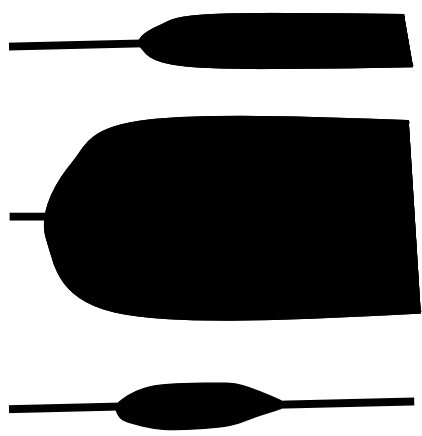

Hypocoagulable state

Hypercoagulable state

Hyperfibrinolytic state

Fig. 4. Principle of global cellular and plasmatic coagulation function tests. Interpretation of thrombelastography traces. a A normal thrombelastography trace, e.g. classic TEG, ROTEM/ ROTEG technologies, allows the interpretation of distinct aspects of cellular coagulation by analysis of several variables. The clotting time $(\mathrm{CT}, \mathrm{r})$ is measured as the time until clotting first starts. The clot formation rate (also designated as $\alpha$ angle, $\alpha$, CFT etc.) is described by the angle measured to the start of fibrin polymerisation. The maximum clot strength (MCF, MA) is described by the thickness of the trace, while the rate of clot lysis can be derived as the maximum lysis, the lysis at a fixed time, or as the maximum lysis. b Pathologic traces show the characteristic findings in hypocoagulable states (prolonged clotting time, decreased clot formation rate and maximum clot strength), hypercoagulable states (shortened clotting time, increased clot formation), and hyperfibrinolytic state (prolonged clotting time, very decreased clot formation rate and maximum clot strength).

lar to the original method, information on clot formation and clot lysis may be obtained.

Recently, rotational TEG (ROTEG/ROTEM, ZLB-Behring) has been introduced, which is a further development of TEG in which the reaction cup is fixed and the needle rotates [44]. On induction of platelet aggregation and coagulation, the restrained movement is recorded. The technique is very flexible and is open to the use of several activators, e.g. ellagic acid (INTEM), rodent tissue factor (EXTEM) or fibrinogen (FIBTEM), and the analysis conditions can be freely applied by the user. Similar to the traditional TEG, in the ROTEM information on several steps of clot formation can be gained: i) the clot formation time determines clot formation dynamics, ii) the $\alpha$-angle determines the kinetics of clot formation, iii) the maximum clot firmness allows a measure of clot stability, and iv) the maximum lysis gives the maximum clot lysis index as a measure of the fibrinolytic activity.

Clot formation can also be measured as platelet contractile force (PCF). Reduced or delayed development of the PCF re- 
sults in structurally weaker clots. Platelet clot forming function and stability is measured in the presence of coagulation factors, particularly thrombin, focussing on the late steps of formation of the platelet clot und building out of the clot retraction to gain stability. Assays addressing this combined activity have been developed [45-47] and have now allowed the development of a commercially available system (Hemostasis Analysis System ${ }^{\circledR}$, Hemodyne Inc.). Agonist-induced clot formation is measured in blood trapped between two parallel surfaces, and the resulting pulling force is detected. This system allows the analysis of the complex interactions of platelets and coagulation factors by providing information on i) the PCF, ii) the clot elastic mode (CEM), and iii) the thrombin generation time (TGT). The TGT is derived from the PCF kinetics curve. In the absence of thrombin, no PCF can be measured. In fact, the initial rise in the PCF curve occurs at the moment of thrombin production. The combined analysis of haemostasis and platelet clot formation may employ PRP or whole blood, but approximately $1 \mathrm{ml}$ of sample blood is required.

Caveats: The PCF is influenced by the platelet number, the expression of GpIIb/IIIa, drugs influencing platelet metabolism and the presence of antithrombin activities. Besides being sensitive to the platelet number, the CEM is also influenced by the concentration of fibrinogen, the rate of thrombin generation and by erythrocyte defects. TGT is sensitive to clotting factor deficiencies and/or inhibitors and antithrombin activity. Thus the TGT is prolonged in haemophilic states and in the presence of coagulation factor inhibitors such as in multiple myeloma. Some inherited platelet disorders such as the Quebec platelet disorder (QPD) are characterised by delayedonset bleeding and increased expression and storage of active urokinase-type plasminogen activator in platelets. The associated 'gain-of-function' abnormalities that increase the lysis of forming or preformed clots, may be analysed by determining the accelerated fibrinolysis which is an important contributor to QPD bleeding. Although the PCF correlates well with platelet-thrombin integrity and functional activity [48]), increases of the PCF have no influence on the total blood loss in an animal model [49], thus compromising the potential clinical relevance. The positive predictive value of abnormal TEG/ ROTEG results on the perioperative blood loss in cardiac surgery is very limited [50].

\section{Global Haemostasis Tests Addressing Platelet Function}

The first in vivo test to analyse platelet function which is still employed as the classic primary screening test for primary haemostasis is the bleeding time.

Principle: The bleeding time is measured, after applying a standardised blood pressure by using a device for standarddepth incisions, as the time required for the stop of spontaneous bleeding (2-10 $\mathrm{min}$ ). Theoretically, the bleeding time represents the natural functional interaction entity of platelets, endothelium, further interacting resident vascular cells and different cell types in the circulation as well as coagulation factors in the three dimensional vessel, still it is neither very specific for the diagnosis of functional platelet defects nor does it have an adequate sensitivity.

Caveats: The bleeding time is dependent on the concentration and function of platelets, on the fibrinogen concentration and on the presence of abnormal fibrinogens (dysfibrinogenaemia) and vascular factors. The bleeding time has a limited reproducibility, and the results are biased by a multitude of interfering conditions, including the skills of the person who performs the test, local skin conditions, etc. In addition to the invasiveness of the test, the method is time-consuming and produces high costs by long hands-on time of the personnel. A major restriction is the lack of correlation between the bleeding time and the bleeding tendency, precluding an adequate use as a screening test to predict the risk of haemorrhage for a given patient [51, 52]. Conversely, the bleeding time is neither predictive for the risk of thrombotic events, as shown in the Caerphilly Prospective Study for ischemic stroke and myocardial infarction [53].

The platelet function analyser (PFA-100 ${ }^{\circledR}$, Dade Behring) as the first commercially available device for the measurement of the in vitro bleeding time was introduced more than 10 years ago.

Principle and use: Anticoagulated blood (usually citrate) is aspirated under standardised shear conditions into disposable cartridges containing membranes precoated with collagen and either ADP or epinephrine as platelet activators. The resulting activation and induction of aggregation of the platelets in whole blood is measured as the time to formation of a platelet plug. The test is simple to perform, rapid (closure times up to a maximum of $300 \mathrm{~s}$ ) but requires relatively large amounts of whole blood $(\sim 1 \mathrm{ml})$ per test, is relatively expensive, and the plastic cartridges are not reusable. The PFA-100 closure time is abnormal in some forms of platelet disorders. It is particularly suited to detect von Willebrand's disease and is more sensitive than the in vivo bleeding time $[54,55]$. The PFA-100 is not uniformly sensitive to detect several other functional platelet abnormalities, including mild type-I von Willebrand's disease, storage pool diseases, primary platelet secretion defects, Hermansky-Pudlak syndrome as well as FV Quebec, and false-negative results may obscure diagnosis. The PFA100 assay is not suited to detect coagulation defects such as haemophilia, isolated coagulation factor deficiencies affecting e.g. FV, FVII, FXI or FXII, or afibrinogenaemia. Although the negative predictive value is relatively high $(>90 \%)$, falsepositive test results occur. Thus an isolated pathologic closure time is not diagnostic. The suitability for predicting bleeding or thrombotic events and aspirin responsivenes [56-59] remains to be conclusively documented.

Caveats: Although the test appears to have a good inter-laboratory reliability, no universal normal range is applicable, and the normal range should be determined for each agonist in 
each laboratory with a panel of normal donors. The assay is particularly sensitive to several factors influencing platelet numbers or function, which may obscure reasonable results. The assay is not reliable in thrombocytopenia with platelet numbers below 100,000/ $\mu$ l and anaemia with a haematocrit < $30 \%$. Moreover, the PFA-100 test is very sensitive to many medications and dietary factors, which influence platelet function. The current studies show that the PFA-100 test does not have sufficient sensitivity and specificity to be used as a screening test for platelet disorders, nor to allow a therapeutic monitoring of platelet function [60].

\section{Platelet Microparticles}

The platelet activation process generates abundant microparticles which exert procoagulant activity and play an increasingly recognised role in haemostasis and are involved in several prothrombotic disorders, including immune thrombocytopenia, and heparin-induced thrombocytopenia. Platelet microparticles occur as budding parts of the platelet membrane or as exosomes. While membrane-derived microparticles range from 0.1 to $1 \mu \mathrm{m}$, exosomes are much smaller (range 40-100 nm) [61]. Recent data have shown that these active microparticles, while containing many well-characterised platelet proteins, embody a range of several hundred unique proteins which had not previously been described in platelets [62] These results suggest that supplementary potentially pathophysiological and clinically relevant information may be obtained by analysis of platelet microparticles in addition to platelet function tests.

\section{Platelet Proteome Analysis and Transcriptomics}

The analysis of the platelet proteome allows a comprehensive and efficient analysis of the protein content and its dynamic changes during functional activation.

Principle and use: Platelets are isolated from whole blood by different precipitation techniques, e.g. by ethanol or tricholoracetic acid precipitation, and the protein profile is usually determined by mass spectrometry in combination with several separation techniques such as two-dimensional gel electrophoresis or multidimensional liquid chromatography $[62,63]$. Proteome analysis is particularly suitable for platelets because of the absence of a nucleus. Recently, the proteome and peptidome of static platelets has been successfully characterised in a basal state. Moreover, the technique has been applied to analyse functional changes upon platelet activation, and the analysis of the signalling cascades in human platelets has allowed the identification of novel platelet signalling proteins and phosphorylation events. In addition, changes in intracellular $\mathrm{Ca}^{2+}$ concentrations with transcriptional activity can be monitored by flow cytometry. By analysing the functional proteome, new insights into the mechanisms of platelet activation can be gained, which may also serve as a basis for the development of therapeutic agents for thrombotic disease. Today, proteomic analysis starts to elucidate rather specific findings for selected platelet functional disorders: For example, low protein tyrosine phosphorylation was identified to characterise platelets in patients with Glanzmann's thrombasthenia [62].

Caveats: The protein profile identified is critically dependent on the methods used to isolate and to prepare the protein samples and varies considerably with the protein precipitation method used. The time delay until processing and the type of anticoagulant used may influence mass spectrometric results. Further important criteria to ascertain reproducibility include storage of the sensitive isolated proteins as well as the use of protease inhibitors during processing. A much simpler approach to analyse the transcriptional activity can be performed by flow cytometry, using $\mathrm{Ca}^{2+}$ ionophores, measurement of intracellular $\mathrm{Ca}^{2+}$ flows and intracellular phosphorylation events in platelets [28]. The technique has only limited applications in the characterisation of platelet integral membrane proteins and surface receptors, due to the low solubility of these proteins.

\section{Platelet Genome Analysis}

Traditionally, platelets were viewed as anucleated cells, lacking nuclear DNA and containing only gradually declining remnants of megakaryocytic mRNA. This presumption has already been challenged in the past by findings of chemokine mRNA, and now signal-depending mRNA splicing has been identified in platelets [3]. Thereby, platelets may actively respond to activation by altering the repertoire of transcription and signal transduction. Pre-mRNA splicing is a unique feature of platelets which has not been observed in any other cell type and which allows splicing of mRNA in the cytoplasm at specific sites, the 'spliceosomes'.

Principle and use: Profiling of platelet transcriptional activity can be addressed by closed global molecular tests such as microarrays containing known DNA probes, allowing to bind and quantify platelet RNA, or by using open screening platforms such as serial analysis of gene expression methods, allowing the detection of unknown RNA. Thereby static and activation-dependent changes of the platelet transcriptome may be described.

Caveats: The major limitation of platelet transcriptome analysis results from the excruciatingly low amount of platelet RNA compared to the vast abundance of leucocyte RNA in peripheral blood. Therefore, the platelet isolation methods ascertaining the purity and leucocyte freeness are central critical points, besides the reproducibility and reliability of the profiling of low-abundance transcripts. 


\section{Platelet Marker Mutation Screening}

The currently known mutations of several genes involved in specific functional platelet disorders, including GpIb, GpIX and GPV as well as NMMHCIIA and GATA-1, can be detected by nucleic acid testing. For the GpIb-V-IX complex which binds vWF, gene defects have been identified for three of the four genes encoding the four subunits of the receptor, GPIBA, GPIBB, GP5 and GP9 (chromosomes 17p12, 22q 11.2, 3q29, and 3q21, respectively) [64]. Only for GP5, so far no defect has been associated with an inherited functional deficiency in humans.

\section{Pre- and Peri-Analytical Considerations}

Platelets probably represent the most reactive cell type in the body. Therefore, peri-analytical handling bears a high risk of artificial activation and deactivation. Since platelet function is critically dependent on the ionised calcium concentration, the use of anticoagulants with different calcium chelation capacity, severely affect the results. Besides the availability of free calcium, the temperature, the venipuncture procedure, the syringe material, the manipulation and handling as well as the age of the sample may contribute to artificial results.

\section{Standardisation and Quality Control}

The main problem of all cell-based functional coagulation tests is the lack of appropriate internal or external quality control reagents and standards. Most assays which differentially address selected platelet functions still remain poorly standardised. This particularly applies to the different variants of aggregometry, but even more so to the bleeding time. In addition, most platelet function tests are performed with fresh blood, which generally requires the parallel assay of known normal control samples, the importance of which is often overlooked.

\section{Rational Diagnostic Algorithm to Identify Platelet Dysfunctions}

The first and most important step of platelet function analysis constitutes of a thorough patient and family history, including a questionnaire for drugs influencing cellular coagulation and a sound physical examination for signs of cellular versus plasmatic coagulation abnormalities. Primary haemostatic disorders are typically characterised by immediate, often disproportionate bleeding following trauma with a mucocutaneous bleeding pattern with petechiae, commonly with menorrhagia, and epistaxis.

The second step addresses the exclusion of frequent abnor- malities, presenting with similar phenotypes as platelet functional disorders. Initial work-up should thus always exclude (concomitant) plasmatic coagulation defects, and thus involve the analysis of PTT, PT and TT. Importantly, von Willebrand's disease as the most common bleeding diathesis has to be excluded since the patients present with similar bleeding symptoms as in platelet dysfunction. Thus, vWF antigen level, FVIII levels, vWF ristocetin cofactor activity and fibrinogen determinations as well as functional vWF assays should be performed to exclude defective platelet adhesion secondary to qualitative or quantitative vWF defects. Hyperfibinolysis in suggestive clinical conditions, e.g. pregnancy, as well as deficiency of FXIII should be considered in differential diagnosis. Secondary functional platelet defects due to drugs or antiplatelet medication [65] or due to underlying disease, particularly myeloproliferative syndromes or liver and renal diseases [9], should be excluded.

In the stepwise diagnostic approach to suspected platelet abnormalities, only the third step actually involves differentiated analysis of platelets. As a general and simple initial investigation a complete blood count with thick film evaluation already allows the identification of the basic quantitative and some qualitative parameters, including thrombocytosis, thrombocytopenia, size variations (e.g. megaplatelets), granule content as well as the exclusion of artificial platelet aggregates (e.g. pseudothrombocytopenia, cold reactive agglutinins). Newer haematological analysers allow the quantification of reticulated platelets and MPV as a means of the thrombopoietic efficiency. The classic and newer direct platelet function tests actually constitute the last step of the diagnostic approach. An overview of the currently employed methods in Germanspeaking countries is provided by a another review of this issue [66]. Due to the restraints of the single platelet function tests described, baseline assays for detecting most functional platelet defects should always constitute of a combination of tests addressing several aspects of platelet function by distinct methods: e.g. aggregometry plus flow cytometry. The bleeding time is no longer recommended for use as baseline screening test because of its non-specificity and lack of clinical correlation. The MPV is simple and cheap to perform and is suggestive for the rare genetic forms with macrothrombocytes often in the presence of thrombocytopenia. Currently the 'gold standard' screening test for platelet function is flow cytometry as the most versatile technology. The turbidimetric platelet aggregometry is also frequently used, but its diagnostic value is limited because of its poor reproducibility and also because of the time-consuming sample preparation and extensive interpretation requirements. If the aggregation abnormalities suggest a secretion or dense granule problem, these should be analysed by additional complex methodologies, combining several aspects of platelet activation and degranulation, such as multiparametric flow cytometry or a combination of procedures such as aggregometry with different platelet agonists plus flow cytometry. The determination of reticulated platelets 
allows differentiation between hypo- and hyperregenerative or reactive thrombocytopenia. Platelet secretion and/or dense granules should also be evaluated if a platelet-type bleeding disorder is suspected but not diagnosed by initial tests. These are mostly diagnosed by exclusion. If no abnormalities are found with tests addressing different steps of platelet activation but the patient history is suggestive, tests for rarer disorders (e.g., Scott's syndrome) should be considered.
In summary, although many new platelet function tests have become available in the last years and several variations of established tests, particularly of flow cytometric assays, have been further developed and their clinical use confirmed, no single test addressing platelet function is capable to cover the broad array of primary and secondary platelet function abnormalities and is suited for monitoring directed anti-platelet therapy.

\section{References}

1 Jurk K, Kehrel BE: Platelets: physiology and biochemistry: Semin Thromb Hemost 2005;31(4): 381-92.

2 Gear AR, Camerini D: Platelet chemokines and chemokine receptors: linking hemostasis, inflammation, and host defense: Microcirculation 2003;10 (3-4):335-50.

3 Denis MM, Tolley ND, Bunting M, Schwertz H, Jiang H, Lindemann S, Yost CC, Rubner FJ, Albertine KH, Swoboda KJ, Fratto CM, Tolley E, Kraiss LW, McIntyre TM, Zimmerman GA, Weyrich AS Escaping the nuclear confines: signal-dependent pre-mRNA splicing in anucleate platelets. Cell 2005;122(3):379-91.

4 Veljkovic DK, Cramer EM, Alimardani G, Fichelson S, Masse JM, Hayward CP: Studies of $\alpha$-granule proteins in cultured human megakaryocytes. Thromb Haemost 2003;90(5):844-52.

$\checkmark 5$ Klouche M, Klinger MH, Kühnel W, Wilhelm D: Endocytosis, storage, and release of IgE by human platelets: differences in patients with type I allergy and nonatopic subjects. J Allergy Clin Immunol 1997;100(2):235-41.

6 Schenk BI, Petersen F, Flad HD, Brandt E: Platelet-derived chemokines CXC chemokine ligand (CXCL)7, connective tissue-activating peptide III, and CXCL4 differentially affect and cross-regulate neutrophil adhesion and transendothelial migration. J Immunol 2002;169(5):2602-10.

$\checkmark 7$ Wilhelm D, Klouche M, Fiebelkorn A, Görg S, Klüter H, Kirchner H: Non-haemolytic transfusion reactions after platelet substitution. Lancet 1993; 342(8867):364.

8 Klinger MH, Jelkmann W: Role of blood platelets in infection and inflammation. J Interferon $\mathrm{Cy}$ tokine Res 2002;22(9):913-22.

9 Jurk K, Kehrel BE : Inherited and acquired disorders of platelet function. Transfus Med Hemother 2007;DOI 10.1159/000098178.

10 Diamandis M, Adam F, Kahr WH, Wang P, Chorneyko KA, Arsenault AL, Rivard GE, Hayward $\mathrm{CP}$ : Insights into abnormal hemostasis in the Quebec platelet disorder from analyses of clot lysis. J Thromb Haemost 2006;4(5):1086-94.

11 Lanza F: Bernard-Soulier syndrome (Hemorrhagiparous thrombocytic dystrophy). Orphanet J Rare Dis 2006;1:46.

12 Harrison P: Platelet function analysis. Blood Rev 2005;19(2):111-23

13 Rothe G: Differential diagnosis of platelet disorders. Dtsch Med Wochenschr 2006;131(5):219-22.

14 Cardigan R, Turner C, Harrison P: Current methods of assessing platelet function: relevance to transfusion medicine. Vox Sang 2005;88(3):153-63.
Campbell PJ, Scott LM, Buck G, Wheatley K, East CL, Marsden JT, Duffy A, Boyd EM, Bench AJ, Scott MA, Vassiliou GS, Milligan DW, Smith SR, Erber WN, Bareford D, Wilkins BS, Reilly JT, Harrison CN, Green AR; United Kingdom Myeloproliferative Disorders Study Group; Medical Research Council Adult Leukaemia Working Party; Australasian Leukaemia and Lymphoma Group: Definition of subtypes of essential thrombocythaemia and relation to polycythaemia vera based on JAK2 V617F mutation status: a prospective study. Lancet 2005;366(9501):1945-53.

16 Briggs CJ, Machin SJ: Discrepancy between impedance and immunofluorescence platelet counting has implications for clinical decision making in patients with idiopathic thrombocytopenia purpura. Br J Haematol 2006;135: 416-417.

17 Segal HC, Briggs C, Kunka S, Casbard A, Harrison P, Machin SJ, Murphy MF: Accuracy of platelet counting haematology analysers in severe thrombocytopenia and potential impact on platelet transfusion. Br J Haematol 2005;128(4):520-5.

18 Butkiewicz AM, Kemona H, Dymicka-Piekarska V, Matowicka-Karna J, Radziwon P, Lipska A: Platelet count, mean platelet volume and thrombocytopoietic indices in healthy women and men. Thromb Res 2006;118(2):199-204.

19 Kienast J, Schmitz G: Flow cytometric analysis of thiazole orange uptake by platelets: a diagnostic aid in the evaluation of thrombocytopenic disorders. Blood 1990 1;75(1):116-21.

20 Abe Y, Wada H, Sakakura M, Nishioka J, Tomatsu H, Hamaguchi Y, Oguni S, Shiku H, Nobori T: Usefulness of fully automated measurement of reticulated platelets using whole blood. Clin Appl Thromb Hemost 2005;11(3):263-70.

21 Ryningen A, Apelseth T, Hausken T, Bruserud O: Reticulated platelets are increased in chronic myeloproliferative disorders, pure erythrocytosis, reactive thrombocytosis and prior to hematopoietic reconstitution after intensive chemotherapy. Platelets 2006;17(5):296-302.

22 Ault KA, Rinder HM, Mitchell J, Carmody MB, Vary CP, Hillman RS: The significance of platelets with increased RNA content (reticulated platelets). A measure of the rate of thrombopoiesis. Am J Clin Pathol 1992;98(6):637-46.

23 Chaoui D, Chakroun T, Robert F, Rio B, Belhocine R, Legrand O, Salanoubat C, Lecrubier C, Casadevall N, Marie JP, Elalamy I: Reticulated platelets: a reliable measure to reduce prophylactic platelet transfusions after intensive chemotherapy. Transfusion 2005;45(5):766-72.
4 McCabe DJ, Harrison P, Sidhu PS, Brown MM, Machin SJ: Circulating reticulated platelets in the early and late phases after ischaemic stroke and transient ischaemic attack. Br J Haematol 2004;126 (6):861-9.

25 Becchi C, Al Malyan M, Fabbri LP, Marsili M, Boddi V, Boncinelli S: Mean platelet volume trend in sepsis: is it a useful parameter? Minerva Anestesiol 2006;72(9):749-56.

26 Gohda F, Uchiumi H, Handa H, Matsushima T, Tsukamoto N, Morita K, Amagai H, Murakami M, Murakami H, Nojima Y, Karasawa M: Identification of inherited macrothrombocytopenias based on mean platelet volume among patients diagnosed with idiopathic thrombocytopenia. Thromb Res 2006; DOI 10.1016/j.thromres.2006.06.011.

27 Bowles KM, Cooke LJ, Richards EM, Baglin TP Platelet size has diagnostic predictive value in patients with thrombocytopenia. Clin Lab Haematol 2005;27(6):370-3.

28 Schmitz G, Rothe G, Ruf A, Barlage S, Tschope D Clemetson KJ, Goodall AH, Michelson AD, Nurden AT, Shankey TV: European Working Group on Clinical Cell Analysis: Consensus protocol for the flow cytometric characterisation of platelet function. Thromb Haemost 1998;79(5):885-96.

29 Linden MD, Frelinger AL 3rd, Barnard MR, Przyklenk K, Furman MI, Michelson AD: Application of flow cytometry to platelet disorders. Semin Thromb Hemost 2004;30(5):501-11.

30 Michelson AD: Evaluation of platelet function by flow cytometry. Pathophysiol Haemost Thromb 2006;35(1-2):67-82.

31 Israels SJ, Gerrard JM, Jacques YV, McNicol A Cham B, Nishibori M, Bainton DF: Platelet dense granule membranes contain both granuloplysin and P-selectin (GMP-140). Blood 1992;80(1):143-52.

32 Ruf A, Patscheke H: Flow cytometric detection of activated platelets: comparison of determining shape change, fibrinogen binding, and P-selectin expression. Semin Thromb Hemost 1995;21(2): 146-51.

33 Barlage S, Wimmer A, Pfeiffer A, Rothe G, Schmitz G: MK-383 (tirofiban) induces a GPIIb/ IIIa receptor conformation which differs from the resting and activated receptor. Platelets 2002;13(3): $133-40$

34 Barnard MR, Linden MD, Frelinger AL III, Li Y, Fox ML, Furman MI, Michelson AD: Effects of platelet binding on whole blood flow cytometry assays of monocyte and neutrophil procoagulant activity. J Thromb Haemost 2005;3(11):2563-70.

35 Gobbi G, Mirandola P, Tazzari PL, Ricci F, Caimi L, Cacchioli A, Papa S, Conte R, Vitale M: Flow cytometry detection of serotonin content and release in resting and activated platelets. Br J Haematol 2003;121(6):892-6. 
36 Fröhlich D, Rothe G, Schmitz G, Hansen E: Volatile anaesthetics induce changes in the expression of P-selectin and glycoprotein Ib on the surface of platelets in vitro. Eur J Anaesthesiol 1998; 15(6):641-8.

37 Wahba A, Rothe G, Lodes H, Barlage S, Schmitz G, Birnbaum DE: Effects of extracorporeal circulation and heparin on the phenotype of platelet surface antigens following heart surgery. Thromb Res 2000;97(6):379-86.

38 Lösche W, Heptinstall S: Value of platelet activation markers as prothrombotic risk indicators. Transfus Med Hemother 2007;DOI: 10.1159/ 000097497

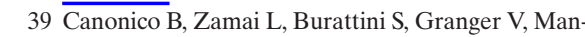
nello F, Gobbi P, Felici C, Falcieri E, Reilly JT, Barnett D, Papa S: Evaluation of leukocyte stabilisation in TransFix-treated blood samples by flow cytometry and transmission electron microscopy. J Immunol Methods 2004;295(1-2):67-78.

40 Wang JC, Aucoin-Barry D, Manuelian D, Monbouquette R, Reisman M, Gray W, Block PC, Block $\mathrm{EH}$, Ladenheim M, Simon DI: Incidence of aspirin nonresponsiveness using the Ultegra Rapid Platelet Function Assay-ASA. Am J Cardiol 2003;92(12): 1492-4.

41 Campbell CL, Berger PB, Nuttall GA, Orford JL, Santrach PJ, Oliver WC, Ereth MH, Thompson CM, Murphy MK, McGlassen DL, Schrader LM, Steinhubl SR: Can N-acetylcysteine reverse the antiplatelet effects of clopidogrel? An in vivo and vitro study. Am Heart J 2005;150(4):796-9.

42 Lennon MJ, Gibbs NM, Weightman WM, McGuire D, Michalopoulos N: A comparison of Plateletworks and platelet aggregometry for the assessment of aspirin-related platelet dysfunction in cardiac surgical patients. J Cardiothorac Vasc Anesth 2004:18(2):136-40.

43 Luddington RJ: Thrombelastography/thromboelastometry. Clin Lab Haematol 2005;27(2):81-90.

44 Lang T, Bauters A, Braun SL, Potzsch B, von Pape KW, Kolde HJ, Lakner M: Multi-centre investigation on reference ranges for ROTEM thromboelastometry. Blood Coagul Fibrinolysis 2005;16(4): 301-10.
45 Topaz O, Minisi AJ, Bernardo NL, McPherson RA, Martin E, Carr SL, Carr ME Jr: Alterations of platelet aggregation kinetics with ultraviolet laser emission: the 'stunned platelet' phenomenon. Thromb Haemost 2001;86(4):1087-93.

46 Carr ME Jr, Martin EJ, Carr SL: Delayed, reduced or inhibited thrombin production reduces platelet contractile force and results in weaker clot formation. Blood Coagul Fibrinolysis 2002;13(3):193-7.

47 Carr ME Jr: Development of platelet contractile force as a research and clinical measure of platelet function. Cell Biochem Biophys 2003;38(1):55-78.

48 Reid TJ, Snider R, Hartman K, Greilich PE, Carr ME, Alving BM: A method for the quantitative assessment of platelet-induced clot retraction and clot strength in fresh and stored platelets. Vox Sang 1998;75(4):270-7.

49 Rothwell SW, Maglasang P, Reid TJ, Gorogias M, Krishnamurti C: Correlation of in vivo and in vitro functions of fresh and stored human platelets. Transfusion 2000;40(8):988-93.

50 Cammerer U, Dietrich W, Rampf T, Braun SL, Richter JA: The predictive value of modified computerized thromboelastography and platelet function analysis for postoperative blood loss in routine cardiac surgery. Anesth Analg 2003;96(1):51-7.

51 Rodgers RP, Levin J: A critical reappraisal of the bleeding time. Semin Thromb Hemost 1990;16(1): $1-20$.

52 Lehman CM, Blaylock RC, Alexander DP, Rodgers GM: Discontinuation of the bleeding time test without detectable adverse clinical impact. Clin Chem 2001;47(7):1204-11.

53 Elwood PC, Pickering J, Yarnell J, O'Brien JR, Ben Shlomo Y, Bath P: Bleeding time, stroke and myocardial infarction: the Caerphilly prospective study. Platelets 2003;14(3):139-41.

54 Fressinaud E, Veyradier A, Truchaud F, Martin I, Boyer-Neumann C, Trossaert M, Meyer D: Screening for von Willebrand disease with a new analyzer using high shear stress: a study of 60 cases. Blood 1998;91(4):1325-31.

55 Posan E, McBane RD, Grill DE, Motsko CL, Nichols WL: Comparison of PFA-100 testing and bleeding time for detecting platelet hypofunction and von Willebrand disease in clinical practice. Thromb Haemost 2003;90(3):483-90.
56 Chakroun T, Gerotziafas G, Robert F, Lecrubier C, Samama MM, Hatmi M, Elalamy I: In vitro aspirin resistance detected by PFA-100 closure time: pivotal role of plasma von Willebrand factor. $\mathrm{Br} \mathrm{J}$ Haematol 2004;124(1):80-5.

57 Andersen K, Hurlen M, Arnesen H, Seljeflot I: Aspirin non-responsiveness as measured by PFA-100 in patients with coronary artery disease. Thromb Res 2002;108(1):37-42.

58 Macchi L, Sorel N, Christiaens L: Aspirin resistance: definitions, mechanisms, prevalence, and clinical significance. Curr Pharm Des 2006;12(2): 251-8.

59 Macchi L, Christiaens L, Brabant S, Sorel N, Allal J, Mauco G, Brizard A: Resistance to aspirin in vitro is associated with increased platelet sensitivity to adenosine diphosphate. Thromb Res 2002;107 (1-2):45-9.

60 Hayward CP, Harrison P, Cattaneo M, Ortel TL, Rao AK, for the Platelet Physiology Subcommittee of the Scientific and Standardization Committee of the International Society on Thrombosis and Haemostasis: Platelet function analyzer (PFA)-100 closure time in the evaluation of platelet disorders and platelet function. J Thromb Haemost 2006;4 (2):312-9.

61 Garcia BA, Smalley DM, Cho H, Shabanowitz J, Ley K, Hunt DF: The platelet microparticle proteome. J Proteome Res 2005;4(5):1516-21.

62 Garcia A: Proteome analysis of signaling cascades in human platelets. Blood Cells Mol Dis 2006;36(2): 152-6.

63 Gnatenko DV, Perrotta PL, Bahou WF: Proteomic approaches to dissect platelet function: half the story. Blood 2006;108(13):3983-91.

64 Lanza F: Bernard-Soulier syndrome (hemorrhagiparous thrombocytic dystrophy). Orphanet J Rare Dis 2006;1:46.

65 Ahrens I, Schwarz M, Peter K, Bode C: Therapeutic inhibitors of platelet aggregation - from aspirin to integrin blockers. Transfusion Med Hemother 2007;DOI 10.1159/000097664

66 Streif W, Olivieri M, Weickardt St, Eberl W, Knoefler R, for the members of the Thromkid study group of the GTH: Thromkid - a competence network for functional platelet abnormalities. Transfusion Med Hemother 2006;DOI 10.1159/000097848. 